\title{
Long-Term Treatment with Empagliflozin Attenuates Renal Damage in Obese Zucker Rat
}

\author{
Authors \\ Nandini D. P. K. Manne1,2, Gautam Kumar Ginjupalli' ${ }^{1}$, Kevin M. Rice ${ }^{1,3}$, Ravikumar Arvapalli ${ }^{1}$, Vincent A. Graffeo ${ }^{4}$, \\ Venkata V. K. Bandarupalli', Eric R. Blough'1,5
}

\section{Affiliations}

1 Center for Diagnostic Nanosystems, Marshall University, Huntington, WV, United States

2 Department of Public Health, Marshall University, Huntington, WV, United States

3 Department of Internal Medicine, Joan C. Edwards School of Medicine, Marshall University, Huntington, WV, USA

4 Department of Pathology, St. Mary's Medical Center, Huntington, WV, United States

5 Department of Pharmaceutical Sciences and Research, Marshall University, Huntington, WV, United States

Key words

SGLT2 inhibitor, type 2 diabetes, glucose tolerance, inflammation

$\begin{array}{ll}\text { received } & 20.07 .2018 \\ \text { revised } & 15.11 .2018 \\ \text { accepted } & 04.12 .2018\end{array}$

\section{Bibliography}

DOI https://doi.org/10.1055/a-0815-4908

Published online: 7.1.2019

Exp Clin Endocrinol Diabetes 2020; 128: 512-519

(C) J. A. Barth Verlag in Georg Thieme Verlag KG Stuttgart .

New York

ISSN 0947-7349

\section{Correspondence}

Nandini D. P. K. Manne, Ph.D.

Room 315, Prichard Hall Department of Public Health

$31718^{\text {th }}$ St. Marshall University

25755 Huntington WV

United States

Tel.: + 1/304/696 3776

manne@marshall.edu

\section{ABSTRACT}

Introduction Empagliflozin, a known inhibitor of sodiumglucose cotransporter type 2 (SGLT2) decreases glucose reabsorption by the renal tubules and promotes glucose excretion into the urine. While the effectiveness of Empagliflozin in the management of hyperglycemia along with associated cardiovascular and all-cause mortality has been demonstrated previously, the therapeutic benefits associated with the long-term use of this drug in obese animals have yet to be investigated.

Methods Male 5-week-old lean and obese Zucker rats were randomly assigned to one of the 4 groups- lean control, lean treated, obese control, obese treated and treated with either Empagliflozin ( $10 \mathrm{mg} / \mathrm{kg} \mathrm{BW} /$ day) or placebo for 25 weeks to investigate the therapeutic effect of Empagliflozin.

Results Empagliflozin treatment in the obese animals was associated with decreased body weight, attenuated the loss of F-actin from the renal tubules and improved renal structure and function. These changes in renal function were associated with significant improvements in the glucose tolerance, and decreased non-fasting circulatory levels of glucose, amylase, and other inflammatory markers including NGAL, cystatin C, and clusterin.

Conclusion Long-term use of Empagliflozin in diabetic obese Zucker rats is associated with improvements in glucose tolerance and decreased loss of renal structure and function.

\section{Introduction}

Type 2 diabetes is a major public health problem and a significant cause of renal failure that contributes to a substantial portion of the disability adjusted life years (DALY). It is primarily characterized by decreased tolerance to glucose and increased resistance to insulin as a result of pancreatic $\beta$-cell dysfunction [1]. With the increase in life expectancy, the costs and deleterious effects of chronic diseases, and diabetes in particular, is on the rise. Indeed, recent estimates project that the incidence of diabetes in the United States will approach 15 cases per 1000 individuals by 2050 [2]. Poor glycemic con- 
trol has been shown to result in several diabetic complications including cardiovascular disease and metabolic syndrome [3]. While the therapeutic choices for management of type 2 diabetes have increased over the past few years, it is noteworthy that there is still a significant proportion of individuals with end stage renal disease due to diabetic nephropathy that require constant care.

Even with increased availability of a large number of blood glucose lowering agents, concerns have been raised with respect to their long-term safety and maintenance of therapeutic efficacy. Sodium-glucose cotransporter 2 (SGLT2) inhibitors are a novel class of drugs that are currently being prescribed for the management of hyperglycemia. Studies have shown that approximately $90 \%$ of glucose reabsorption occurs in $\mathrm{S} 1$ segment of proximal convoluted tubule with SGLT2's more predominantly expressed in the diabetic individuals with exacerbating hyperglycemia [4]. These cotransporters act by transporting glucose uphill against the energy gradient and subsequently result in the reabsorption of glucose. The SGLT2 inhibitors, by inhibiting these co-transporters, cause a decrease in glycemic load. In addition, they have been shown to act via an insulin independent mechanism suggesting that they may be ideal for individuals with comorbidities associated with metabolic deregulation [5].

Several clinical studies have been conducted to investigate the effects of SGLT2 inhibitors for the management of hyperglycemia and renal protection [6-8]. Recent work has demonstrated that SGLT2 inhibition promotes urinary excretion of glucose without renal impairment which appears to be associated with decreased cardiovascular mortality and improved overall quality of life $[9,10]$. Empagliflozin is one such drug that has been shown to confer cardio-renal protection through increased urinary excretion of glucose as well as through reduction in blood pressure and an increase in HDL cholesterol $[10,11]$. In addition to these protective mechanisms, additional studies have suggested that other factors may also play a role in cardio-renal protection. For example, it is thought that hyperglycemia induced glomerular hyperfiltration and subsequent activation of renin-angiotensin-aldosterone system is associated with increased intraglomerular pressure. Skrtic et al., has demonstrated that treatment of diabetic individuals with Empagliflozin resulted in a 6-8 mm reduction in intraglomerular pressure [12]. This is particularly important as increased intraglomerular pressure has been shown to result in glomerular injury [13]. In addition, other studies have also indicated that SGLT2 inhibition is associated with reduction in macroalbuminuria [14], indices of renal fibrosis [15] and inflammation [16]. Whether the beneficial effects of Empagliflozin treatment are maintained with long term use is currently unclear. The purpose of the current study was to investigate the therapeutic effects of long term treatment with Empagliflozin in the diabetic Obese Zucker animal model.

\section{Materials and Methods}

\section{Animals}

All procedures were performed in accordance with Association for Assessment and Accreditation of Laboratory Animal Care (AAALAC) and Institutional Animal Care and Use Committee (IACUC) of Marshall University. 4-week-old male lean Zucker (strain code 186) and obese Zuck- er rats (strain code 185) were purchased from the Charles River Laboratories (Wilmington, MA, USA) and housed 2 per cage in an AAALAC approved vivarium. Housing conditions consisted of a $12 \mathrm{H}: 12 \mathrm{H}$ darklight cycle and temperature was maintained at $22 \pm 2{ }^{\circ} \mathrm{C}$. Animals were provided food and water ad libitum and were allowed to acclimatize for 1 week before any experiments were conducted. Animals were then randomly assigned to one of 4 different groups- lean control (LC), lean treated (LT), obese control (OC), and obese treated (OT) accordingly. Two separate sets of animals were used with 4 groups in each set as mentioned earlier. One set of animals ( $n=5-9 /$ group) were used for experiments associated with glucose tolerance test. The second set of animals ( $n=6-8 /$ group) were used for other experiments as detailed in the subsequent sections. The treated animals received Empagliflozin ( $10 \mathrm{mg} / \mathrm{kg}$ body weight/day) that was kindly supplied by Boehringer Ingelheim (KG, Germany) in drinking water for 25 weeks. The amount of Empagliflozin added to drinking water was adjusted weekly based on body weight and their water consumption from prior week. Food consumption and body weights were measured once per week throughout the study duration.

\section{Intra-peritoneal glucose tolerance test (IPGTT)}

Animals were fasted overnight before performing the IPGTT test. In addition, Empagliflozin was removed from drinking water $\sim 28-32 \mathrm{~h}$ before performing the test to accurately determine the long-term effects of the drug on glucose handling. The time period for fasting was chosen based on the previous studies that have reported the half-life of Empagliflozin in rodents to be $\sim 2 \mathrm{~h}$ [17].

IPGTT was performed by intraperitoneal injection of glucose at the rate of $1.5 \mathrm{~g} / \mathrm{kg}$ body weight. Animals were restrained and blood was collected through a tail nick with 22 gauge needle. Blood glucose levels were measured a using Bayer Contour Next Ez blood glucose monitoring system (Ascensia, NJ, USA) at baseline and after $15,30,60,90$ and 120 min of glucose administration.

\section{Serum biochemical analysis and multiplex assay}

Animals were humanely sacrificed under anesthesia at the chosen time point and blood was collected through cardiac puncture into a BD Vacutainer ${ }^{\circledR}$ tube. Serum was collected by centrifugation of the tubes at $1000 \mathrm{xg}$ for $10 \mathrm{~min}$. Biochemical parameters were determined by using Abaxis VetScan ${ }^{\circledR}$ analyzer (Abaxis, Union City, CA, USA) as described previously [18]. Milliplex multiplex assays were performed to evaluate the changes in inflammatory markers in urine samples according to manufacturer's instructions (EMD Millipore, MA, USA).

\section{Histopathology and staining for F-actin}

Briefly, frozen kidneys were sectioned $(4 \mu \mathrm{m})$ on to poly-L-lysine coated slides using a Leica CM1950 cryostat as described elsewhere [19]. Renal sections were then stained with hematoxylin and eosin, visualized using an Evos XL microscope (Life technologies, Grand Island, NY) and the extent of tubular, interstitial and glomerular injury determined. A score of 0 indicated no damage, 1-moderate damage, and 2- severe damage. Sections were given a cumulative score that ranged between $0-6$. Renal sections were also stained for F-actin using rhodamine phalloidin (Life technologies, Grand Island, NY, USA) as described previously [19]. Images were captured using an Evos FL microscope (Life Technologies, Grand Island, NY, 
USA) and were evaluated for changes in fluorescence intensity across groups using Image J software (NIH, USA).

\section{Statistical analysis}

Data were analyzed using the SigmaPlot v. 12 program (Systat software Inc., San Jose, CA) and the results are presented as mean \pm SEM. Required sample size was calculated using the resource equation method. A one-way analysis of variance (ANOVA) or 2-way repeated measures ANOVA was performed for overall comparisons followed by the appropriate post hoc test to determine significant differences between groups. For non-normally distributed samples, a Kruskal Wallis $h$ test was performed. A p value of $\leq 0.05$ was considered to be statistically significant.

\section{Results}

\section{Effect of long-term empagliflozin treatment on body weight and food intake in the obese Zucker rat}

The effects of Empagliflozin on body weight and food consumption are shown in -Fig. 1,2. Compared to the lean control animals, there was no significant change in body weight of lean animals that had been treated at 5, 10 and 15 weeks of age. However, the lean treated ani-

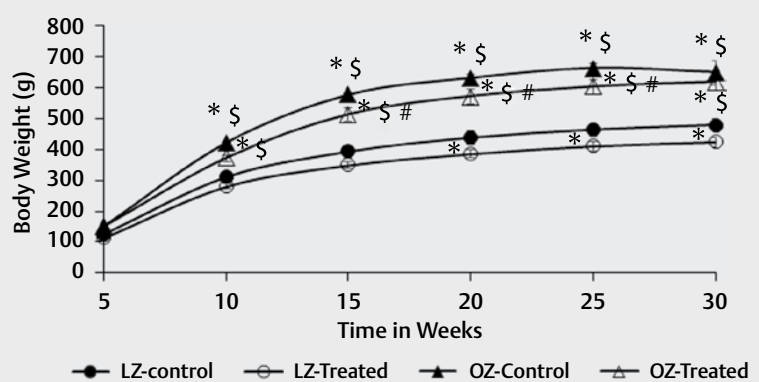

-Fig. 1 Long term treatment with Empagliflozin attenuates body weight gain in Zucker rats. Results are expressed as mean \pm S.E.M. ${ }^{*} p<0.05$ compared with lean control group, $\$ p<0.05$ compared with lean treated group, and \#p<0.05 compared with obese control group ( $n=6-8 /$ group).

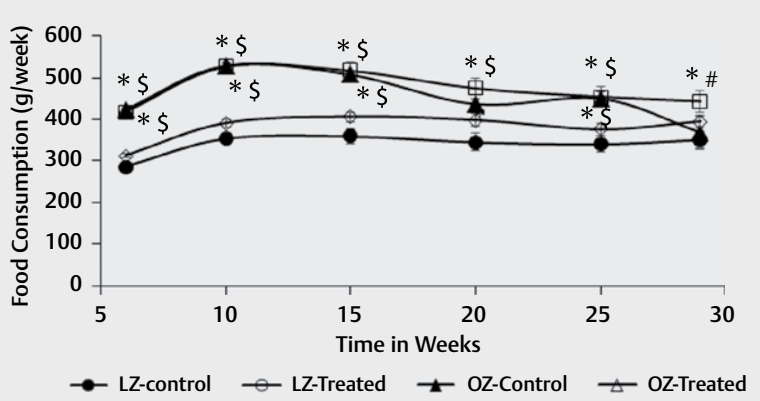

-Fig. 2 Food consumption in Zucker rats treated with Empagliflozin. Results are expressed as mean \pm S.E.M. ${ }^{*} p<0.05$ compared with lean control group, $\$ p<0.05$ compared with lean treated group, and $\# p<0.05$ compared with obese control group ( $n=6-8 /$ group). mals had a significantly lower body weight when compared to their counter parts at 20, 25 and 30 weeks of age ( $/$ Fig. 1 ). Similarly, there was no significant change in the body weight of obese control animals that had been treated at 5, 10 and 30 weeks of age compared to their counter parts. There was a significant decrease in body weight in obese treated animals at 15, 20 and 25 weeks of age (

Compared to lean control group, the lean treated groups did not show a significant change in food consumption ( $>$ Fig. 2). Similarly, the obese treated groups did not show a significant change in food consumption when compared to their counterparts except at 29 weeks of age ( $>$ Fig. 2 ).

\section{Long term empagliflozin treatment attenuates circulating levels of glucose and other biochemical parameters in the obese zucker rat}

Compared to the controls, the lean and obese treated groups did not show a significant change in levels of albumin, globulin, alkaline phosphatase (ALP), blood urea nitrogen (BUN), calcium, phosphorus, sodium, potassium, creatinine, and TBIL. However, the lean treated animals showed a significant decrease in levels of total protein while obese treated animals showed a significant decrease in amylase and glucose levels when compared to their control counterparts ( Table $\mathbf{1}$ ).

\section{Effects of empagliflozin on glucose tolerance in zucker rats}

IPGTT was conducted to investigate the effect of Empagliflozin on long term glucose handling in Zucker rats. Compared to the lean control animals, lean treated animals did not show any significant difference in glucose levels at either 0, 15, 30, 60, 90 and 120 min after glucose administration.

However, between the obese groups, glucose levels were similar at 0,15 and 30 min while at 60 and 90 min after glucose injection, the treated groups showed a significant decrease in circulating glucose levels when compared to the controls demonstrating a significant treatment effect ( $\triangleright$ Fig. $\mathbf{3})$.

\section{Empagliflozin treatment attenuates diabetes- induced renal injury in the obese zucker rat}

The lean treated animals did not exhibit any marked changes in renal structure compared to their counterparts. However, the diabetic obese Zucker animals demonstrated significant proximal tubular dilation with loss of brush border, interstitial inflammation and tubular damage. These changes were significantly attenuated with Empagliflozin treatment ( $\mathbf{F i g}$. 4).

Renal sections were stained with rhodamine phalloidin to evaluate F-actin in tubular cells. Renal sections of obese control animals showed a marked decrease in F-actin staining when compared to the lean control animals. In contrast, treatment with Empagliflozin attenuated the loss of F-actin in the obese treated group ( $\triangleright$ Fig. 5).

\section{Empagliflozin treatment attenuates biochemical markers of renal injury in obese zucker rats}

Multiplex analysis was performed on urine samples to evaluate the effects of long term Empagliflozin treatment on renal damage markers. Compared to the lean control group, the animals in lean treated group showed a slightly significant increase in urinary levels of NGAL and a 
- Table 1 Empagliflozin attenuates diabetes induced alterations in serum biochemical parameters.

\begin{tabular}{|c|c|c|c|c|}
\hline Analyte & 30 WK LZ-control & 30 WK LZ-Treated & 30 WK OZ-Control & 30 WK OZ-Treated \\
\hline Albumin (G/DL) & $3.1 \pm 0.15$ & $3.03 \pm 0.17$ & $3.02 \pm 0.04$ & $2.96 \pm 0.07$ \\
\hline Globulin (G/DL) & $2.07 \pm 0.19$ & $1.84 \pm 0.15$ & $2.82 \pm 0.09 * \$$ & $2.74 \pm 0.13$ \\
\hline Tot. Protein (G/DL) & $5.17 \pm 0.08$ & $4.89 \pm 0.08 *$ & $5.78 \pm 0.05^{* \$}$ & $5.67 \pm 0.10 * \$$ \\
\hline ALT (U/L) & $40.50 \pm 2.74$ & $64.50 \pm 6.28^{*}$ & $37.00 \pm 2.62^{\$}$ & $43.00 \pm 3.91^{\$}$ \\
\hline $\operatorname{ALP}(\mathrm{U} / \mathrm{L})$ & $106.33 \pm 10.91$ & $111.75 \pm 7.42$ & $106.83 \pm 8.2$ & $108.86 \pm 8.65$ \\
\hline Amylase (U/I) & $698.17 \pm 22.75$ & $645.75 \pm 20.89$ & $872.5 \pm 16.02 * \$$ & $803.14 \pm 22.05$ * \$\# \\
\hline BUN (MG/DL) & $18.33 \pm 0.71$ & $17.75 \pm 0.86$ & $35.83 \pm 9.12$ & $35.71 \pm 9.93$ \\
\hline Calcium (MG/DL) & $10.25 \pm 0.06$ & $9.86 \pm 0.10$ & $11.33 \pm 0.16^{\$}$ & $11.54 \pm 0.27^{\$}$ \\
\hline Phosphorus (MG/DL) & $5.65 \pm 0.26$ & $5.71 \pm 0.27$ & $7.08 \pm 0.78$ & $6.97 \pm 0.91$ \\
\hline Sodium (MMOL/L) & $135.00 \pm 0.58$ & $135.88 \pm 0.67$ & $138.00 \pm 1.06^{*}$ & $138.43 \pm 0.75^{*}$ \\
\hline Potassium (MMOL/L) & $4.62 \pm 0.14$ & $4.68 \pm 0.19$ & $5.82 \pm 0.22 * \$$ & $5.30 \pm 0.24^{*}$ \\
\hline Creatinine (MG/DL) & $0.43 \pm 0.06$ & $0.51 \pm 0.04$ & $0.73 \pm 0.15$ & $0.71 \pm 0.20$ \\
\hline Glucose (MG/DL) & $424.50 \pm 10.05$ & $380.38 \pm 9.03$ & $427.67 \pm 25.64$ & $348.43 \pm 9.10$ *\# \\
\hline TBIL (MG/DL) & $0.33 \pm 0.02$ & $0.31 \pm 0.02$ & $0.37 \pm 0.02$ & $0.47 \pm 0.03 * \$$ \\
\hline
\end{tabular}

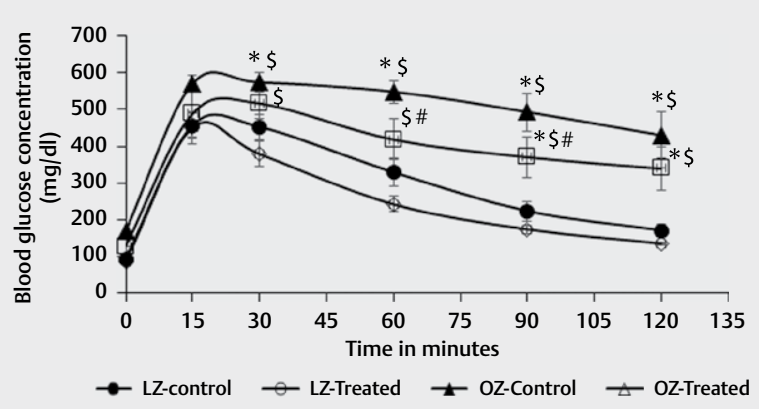

Fig. 3 Long term treatment with Empagliflozin improves glucose tolerance in obese Zucker animals. a) Intra-Peritoneal Glucose Tolerance Test (IPGTT)- Blood glucose concentration in 30 week old Zucker rats. Results are expressed as mean \pm S.E.M. ${ }^{*} p<0.05$ compared with lean control group, $\$ p<0.05$ compared with lean treated group, and \#p $<0.05$ compared with obese control group ( $n=5-9 / g r o u p)$.

decrease in the levels of cystatin C and clusterin (>Fig. 6). In the obese group, treatment significantly attenuated diabetes induced increase in levels of neutrophil gelatinase-associated lipocalin (NGAL), cystatin C and clusterin. Interestingly, the levels of calbindin between lean and obese control did not show any significant difference but the treated groups showed a significant increase when compared to their control counterparts ( Fig. 6).

\section{Discussion}

Type 2 diabetes and its associated cardiovascular complications are a significant cause of morbidity and mortality in the United States [20]. Empagliflozin is a novel SGLT 2 inhibitor that promotes urinary excretion of glucose and is currently prescribed for patient with type 2 diabetes [21]. Previous pre-clinical studies have indicated that short term treatment with Empagliflozin is associated with the attenuation of vascular dysfunction, preservation of $\beta$ cell mass and a significant reduction in albuminuria [22-24]. However, the long-term effects of Empagliflozin treatment have not yet been fully elucidated in similar models. This lack of knowledge is important as certain adverse events are apparent only with prolonged use of the therapeutic agents. While the EMPA-Reg Outcome trial involved treatment of the patients for a median of 2.8 years [10], the current study investigated the effect of treatment for 25 weeks in obese Zucker rats which is approximately equivalent to 12.7 human years [25]. Nonetheless, we advise caution in translating our findings to humans due to significant differences in physiology along with various other confounders. In the current study, we found that long term treatment is associated with a significant reduction in body weight in the treated animals when compared to their counterparts ( $\mathbf{F i g}$. 1). These changes are in agreement with previous studies that reported a decrease in body weight gain with Empagliflozin treatment [26]. These changes in decreased body weight in the obese treated animals were seen despite any significant change in food consumption when compared to the control group over the course of the study ( $\triangleright$ Fig. 2). Although, we did not investigate the correlation between reduced body weight and adiposity with treatment, previous studies have indicated that the treatment was associated with decrease in fat mass as well as other markers of visceral adiposity [27] which were in turn associated with improved renal function [28].

Next, we sought to examine the effect of long term treatment with Empagliflozin on the circulating levels of glucose and other biochemical parameters. Similar to other studies that have shown a reduction in non-fasting blood glucose levels with short term Empagliflozin treatment, we found that the same effect is maintained even with long term treatment ( $\triangleright$ Table 1 ). This is important as certain anti-diabetic drugs have been shown to exhibit a reduction in efficacy with the progression of diabetes [29]. To confirm our findings, we next performed IPGTT to examine the effect of Empagliflozin on glucose tolerance and as expected, treatment with Empagliflozin attenuated obesity induced increase in blood glucose levels over the course of a $2 \mathrm{~h}$ time period when compared with the 
a

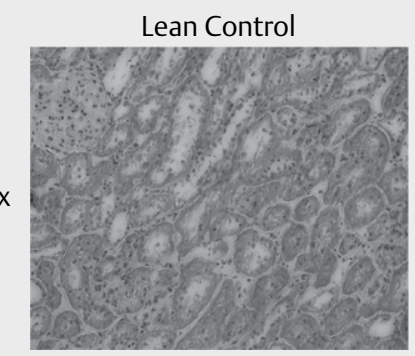

b

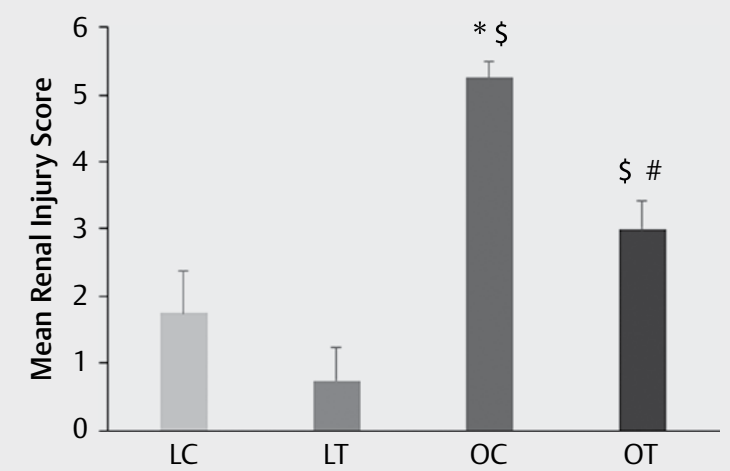

Lean Treated
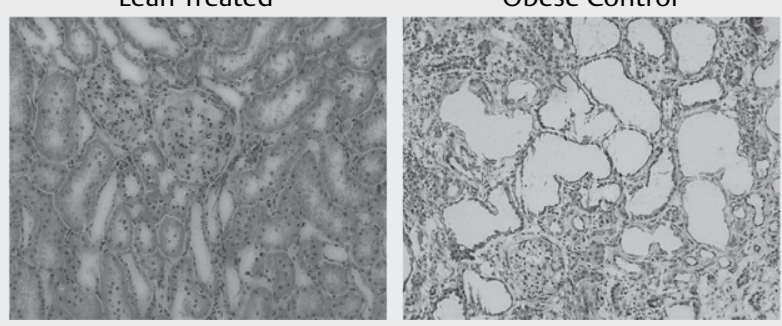

Obese Treated

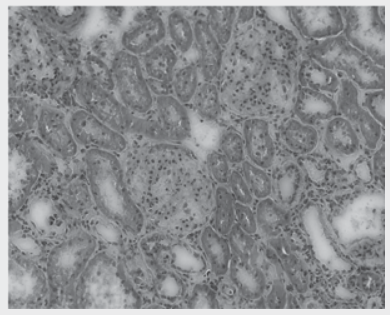

Fig. 4 Effect of Empagliflozin on diabetes induced renal injury. a Hematoxylin and eosin staining of renal sections in 30 week old Zucker rats. b Semi-quantitative histological injury score of renal sections. Results are expressed as mean \pm S.E.M. ${ }^{*} p<0.05$ compared with lean control group, $\$ p<0.05$ compared with lean treated group, and $\# p<0.05$ compared with obese control group ( $n=4 /$ group).

$20 x$

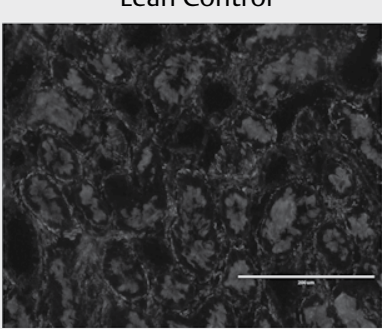

Lean Treated

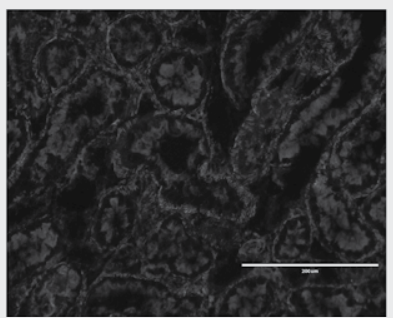

Obese Control

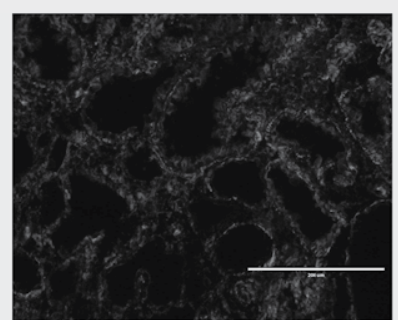

Obese Treated

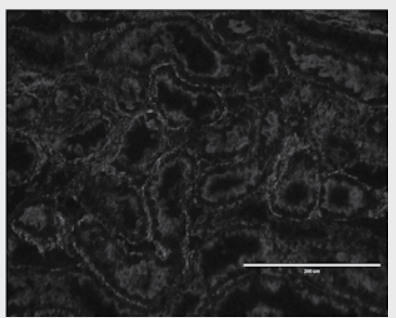

b

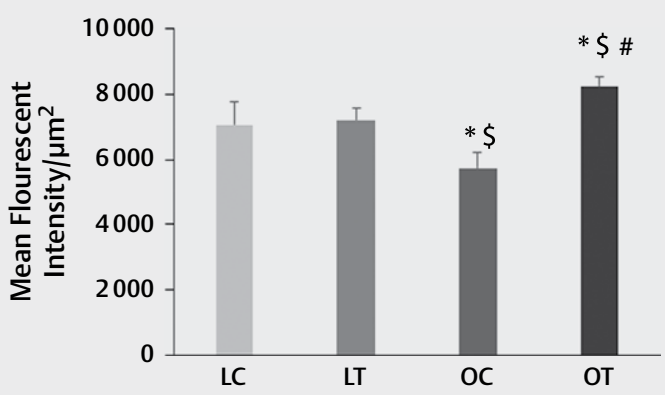

Fig. 5 Effect of Empagliflozin on f-actin loss in Zucker rats. a Rhodamine phalloidin staining for F-actin in renal sections of Zucker rats, $\mathbf{b}$ Mean fluorescence intensity of rhodamine phalloidin sections as a measure of F-actin levels. Results are expressed as mean \pm S.E.M. ${ }^{*} p<0.05$ compared with control group, $\$ p<0.05$ compared with $\mathrm{CeO}_{2}$ group, and \#p<0.05 compared with IR group ( $\mathrm{n}=3 / \mathrm{group}$ ).

untreated counterpart ( Fig. 3 ). While several studies have shown similar findings with short term treatment [30], it is not known if Empagliflozin has a significant effect on blood glucose levels once the drug administration has been withdrawn. Given that the half- life of Empagliflozin to be approximately $2 \mathrm{~h}$ in a rodent model, we withheld drug administration approximately $28-32 \mathrm{~h}$ before measuring the blood glucose levels for IPGTT to determine the true biological effect of the drug. 
a
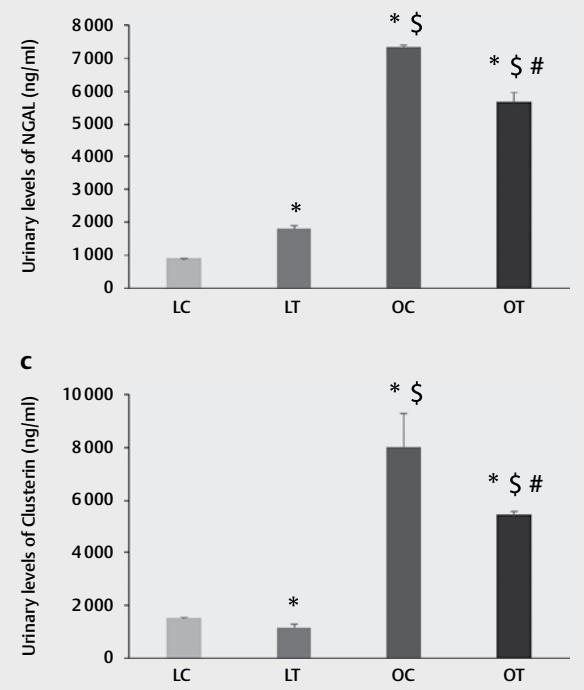

b

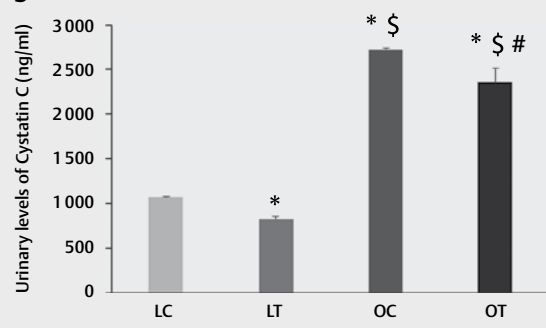

d

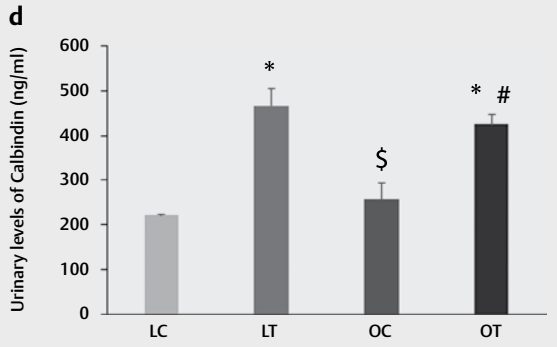

- Fig. 6 Long term treatment with Empagliflozin attenuates markers of renal injury. a Urinary levels of NGAL in 30 week old lean and obese Zucker rats. b Urinary levels of cystatin $C$ in 30 week old lean and obese Zucker rats. c Urinary levels of clusterin in 30 week old lean and obese Zucker rats. d Urinary levels of calbidin in 30 week old lean and obese Zucker rats. Results are expressed as mean \pm S.E.M. ${ }^{*} \mathrm{p}<0.05$ compared with lean control group, $\$ p<0.05$ compared with lean treated group, and \#p<0.05 compared with obese control group ( $n=6 /$ group).

Diabetes is one of the most common causes of cardiovascular diseases, non-traumatic amputations, and end stage renal failure [31-33]. Studies have indicated that diabetes induced renal failure is characterized by 5 stages. Stage 1 and 2 are characterized by renal hypertrophy and increased glomerular filtration rate (GFR) while stages 3-5 are characterized by albuminuria, high blood pressure, reduced GFR and uremia [34]. While several studies have indicated that Empagliflozin attenuated diabetes induced macroalbuminuria [14], increase in intraglomerular pressure [12] and other indices of renal damage [35], it is not known if these effects were maintained with a long term therapy. In the current study, we found that the treatment did not result in an increase in BUN, creatinine and other serum biochemical parameters such as albumin, globulin, alanine aminotransaminase, aspartate transaminase, calcium, sodium, potassium, phosphorus, total bilirubin when compared to their control counterparts ( $\triangleright$ Table 1 ). In addition, Empagliflozin treatment attenuated diabetes-induced increases in renal inflammatory markers such as cystatin C, NGAL and clusterin (• Fig. 6). Interestingly urinary levels of NGAL have been increased significantly in the lean treated rats compared to their counterparts similar to that see in other studies ( $\mathbf{F i g}$. 6) [35]. While the exact cause is yet to be investigated, it could be attributed to increased urine production [35]. NGAL and cystatin C have been shown to be a novel surrogate markers for diabetic nephropathy and associated cardiovascular events [36]. In other studies, cystatin $C$ has been shown to serve as an early biomarker for impaired renal function in comparison with microalbuminuria and serum creatinine levels [37]. Similarly, Kim et al., demonstrated that increased urinary levels of clusterin is associated with early stages of diabetic kidney disease [38]. To further investigate the therapeutic effects of Empagliflozin we sought to investigate the renal structure. Previous stud- ies have shown that increased urinary expression of these inflammatory markers is associated with loss in renal structure [39]. Hematoxylin and eosin staining demonstrated that Empagliflozin treatment attenuated diabetes induced loss of proximal tubule brush border, tubular dilatation and glomerular injury when compared to the control groups ( $\mathbf{F i g}$. 4). These changes were also associated with decreased loss of F-actin in the tubules in the treated animals ( $>$ Fig. 5). Our data supports a recent study by Wanner et al. , that suggested the reno-protective effects of Empagliflozin may be related to decreased proximal tubular sodium reabsorption, decreased intra-glomerular pressure and involvement of neurohormonal systems [40].

In summary, our study demonstrates that long term treatment with Empagliflozin attenuates body weight gain and circulating blood glucose levels and appears to improve renal structure and function without evidence of toxicity in a rodent model of metabolic syndrome. These findings are important as renal failure in individuals with diabetic complications could have devastating effects. The data from our preclinical study highlights the therapeutic benefits of long term treatment with Empagliflozin for the management of type 2 diabetes.

\section{Acknowledgements}

This work was supported in part, by funding from Boehringer-Ingelheim International $\mathrm{GmbH}$ that is awarded to E.R.B. A part of the study was published in the form of an abstract that is presented at Experimental Biology Conference held in Chicago (2017). The author(s) meet criteria for authorship as recommended by the International Committee of Medical Journal Editors (ICMJE). 


\section{Conflict of Interest}

No conflict of interest has been declared by the authors.

\section{References}

[1] Weyer C, Bogardus C, Mott DM et al. The natural history of insulin secretory dysfunction and insulin resistance in the pathogenesis of type 2 diabetes mellitus. J Clin Invest 1999; 104: 787-794

[2] Boyle JP, Thompson T], Gregg EW et al. Projection of the year 2050 burden of diabetes in the US adult population: Dynamic modeling of incidence, mortality, and prediabetes prevalence. Popul Health Metr 2010; 8: 29

[3] Kishore P, Kim SH, Crandall JP. Glycemic control and cardiovascular disease: What's a doctor to do? Curr Diab Rep 2012; 12: 255-264

[4] Kojima N, Williams JM, Takahashi T et al. Effects of a new SGLT2 inhibitor, luseogliflozin, on diabetic nephropathy in T2DN rats. J Pharmacol Exp Ther 2013; 345: 464-472

[5] Vallon V, Thomson SC. Targeting renal glucose reabsorption to treat hyperglycaemia: The pleiotropic effects of SGLT2 inhibition. Diabetologia 2017; 60: 215-225

[6] Chao EC. SGLT-2 Inhibitors: A new mechanism for glycemic control. Clin Diabetes 2014; 32: 4-11

[7] List JF, Woo V, Morales E et al. Sodium-glucose cotransport inhibition with dapagliflozin in type 2 diabetes. Diabetes Care 2009; 32: 650-657

[8] Seidu S, Kunutsor SK, Cos X et al. SGLT2 inhibitors and renal outcomes in type 2 diabetes with or without renal impairment: A systematic review and meta-analysis. Prim Care Diabetes 2018; 12: 265-283

[9] Grandy S, Hashemi M, Langkilde AM et al. Changes in weight loss-related quality of life among type 2 diabetes mellitus patients treated with dapagliflozin. Diabetes Obes Metab 2014; 16: 645-650

[10] Zinman B, Wanner C, Lachin JM et al. Empagliflozin, cardiovascular outcomes, and mortality in type 2 diabetes. N Engl J Med 2015; 373 : 2117-2128

[11] Abdul-Ghani M, Del Prato S, Chilton R et al. SGLT2 Inhibitors and cardiovascular risk: Lessons learned from the empa-reg outcome study. Diabetes Care 2016; 39: 717-725

[12] Skrtic M, Yang GK, Perkins BA et al. Characterisation of glomerular haemodynamic responses to SGLT2 inhibition in patients with type 1 diabetes and renal hyperfiltration. Diabetologia 2014; 57: 2599-2602

[13] Tonneijck L, Muskiet MH, Smits MM et al. Glomerular hyperfiltration in diabetes: Mechanisms, clinical significance, and treatment. J Am Soc Nephrol 2017; 28: 1023-1039

[14] Cherney DZI, Zinman B, Inzucchi SE et al. Effects of empagliflozin on the urinary albumin-to-creatinine ratio in patients with type 2 diabetes and established cardiovascular disease: An exploratory analysis from the EMPA-REG OUTCOME randomised, placebo-controlled trial. Lancet Diabetes Endocrinol 2017; 5: 610-621

[15] Abbas NAT, El Salem A, Awad MM. Empagliflozin, SGLT2 inhibitor, attenuates renal fibrosis in rats exposed to unilateral ureteric obstruction: Potential role of klotho expression. Naunyn Schmiedebergs Arch Pharmacol 2018; 391: 1347-1360

[16] Wang XX, Levi J, Luo $Y$ et al. SGLT2 protein expression is increased in human diabetic nephropathy: SGLT2 protein inhibition decreases renal lipid accumulation, inflammation, and the development of nephropathy in diabetic mice. J Biol Chem 2017; 292: 5335-5348

[17] Grempler R, Thomas L, Eckhardt M et al. Empagliflozin, a novel selective sodium glucose cotransporter-2 (SGLT-2) inhibitor: Characterisation and comparison with other SGLT-2 inhibitors. Diabetes Obes Metab 2012; 14: 83-90
[18] Nalabotu SK, Kolli MB, Triest WE et al. Intratracheal instillation of cerium oxide nanoparticles induces hepatic toxicity in male spraguedawley rats. Int J Nanomedicine 2011; 6: 2327-2335

[19] Manne ND, Arvapalli R, Nepal $\mathrm{N}$ et al. Cerium oxide nanoparticles attenuate acute kidney injury induced by intra-abdominal infection in Sprague-Dawley rats. Journal of nanobiotechnology 2015; 13: 75

[20] Leon BM, Maddox TM. Diabetes and cardiovascular disease: Epidemiology, biological mechanisms, treatment recommendations and future research. World J Diabetes 2015; 6: 1246-1258

[21] Ndefo UA, Anidiobi NO, Basheer E et al. Empagliflozin (Jardiance): A novel SGLT2 Inhibitor for the treatment of type-2 diabetes. P T 2015; 40: $364-368$

[22] Cheng ST, Chen L, Li SY et al. The effects of empagliflozin, an SGLT2 Inhibitor, on Pancreatic beta-cell mass and glucose homeostasis in type 1 diabetes. PLoS One 2016; 11: e0147391. doi:10.1371/journal. pone. 0147391

[23] Oelze M, Kroller-Schon S, Welschof P et al. The sodium-glucose co-transporter 2 inhibitor empagliflozin improves diabetes-induced vascular dysfunction in the streptozotocin diabetes rat model by interfering with oxidative stress and glucotoxicity. PLoS One 2014; 9: e112394. doi:10.1371/journal.pone.0112394

[24] Vallon V, Gerasimova M, Rose MA et al. SGLT2 inhibitor empagliflozin reduces renal growth and albuminuria in proportion to hyperglycemia and prevents glomerular hyperfiltration in diabetic Akita mice. Am J Physiol Renal Physiol 2014; 306: F194-F204

[25] Sengupta P. The laboratory rat: Relating its age with human's. Int J Prev Med 2013; 4: 624-630

[26] Steven S, Oelze M, Hanf A et al. The SGLT2 inhibitor empagliflozin improves the primary diabetic complications in ZDF rats. Redox Biol 2017; 13: 370-385

[27] Neeland IJ, McGuire DK, Chilton R et al. Empagliflozin reduces body weight and indices of adipose distribution in patients with type 2 diabetes mellitus. Diab Vasc Dis Res 2016; 13: 119-126

[28] Young JA, Hwang S], Sarnak MJ et al. Association of visceral and subcutaneous adiposity with kidney function. Clin J Am Soc Nephrol 2008; 3: 1786-1791

[29] Chaudhury A, Duvoor C, Reddy Dendi VS et al. Clinical review of antidiabetic drugs: Implications for type 2 diabetes mellitus management. Front Endocrinol (Lausanne) 2017; 8: 6

[30] Hansen HH, Jelsing J, Hansen CF et al. The sodium glucose cotransporter type 2 inhibitor empagliflozin preserves beta-cell mass and restores glucose homeostasis in the male zucker diabetic fatty rat. J Pharmacol Exp Ther 2014; 350: 657-664

[31] Grundy SM, Benjamin IJ, Burke GL et al. Diabetes and cardiovascular disease: A statement for healthcare professionals from the american heart association. Circulation 1999; 100: 1134-1146

[32] Apelqvist J, Larsson J. What is the most effective way to reduce incidence of amputation in the diabetic foot? Diabetes/Metabolism Research and Reviews 2000; 16: S75-S83

[33] Narres M, Claessen H, Droste $\mathrm{S}$ et al. The incidence of end-stage renal disease in the diabetic (compared to the non-diabetic) population: A systematic review. PLoS One 2016; 11: e0147329. doi:10.1371/ journal.pone. 0147329

[34] Mogensen CE, Christensen CK, Vittinghus E. The stages in diabetic renal disease. With emphasis on the stage of incipient diabetic nephropathy. Diabetes 1983; 32: (Suppl 2): 64-78

[35] Gallo LA, Ward MS, Fotheringham AK et al. Once daily administration of the SGLT2 inhibitor, empagliflozin, attenuates markers of renal fibrosis without improving albuminuria in diabetic $\mathrm{db} / \mathrm{db}$ mice. Sci Rep 2016; 6: 26428 
[36] Alter ML, Kretschmer A, Von Websky K et al. Early urinary and plasma biomarkers for experimental diabetic nephropathy. Clin Lab 2012; 58 : 659-671

[37] Ascic-Buturovic B, Cavaljuga S. Cystatin C as a marker for detection of early renal failure in diabetes type 2 patients. Bosn J Basic Med Sci 2005; 5: 68-71

[38] Kim SS, Song SH, Kim JH et al. Urine clusterin/apolipoprotein J is linked to tubular damage and renal outcomes in patients with type 2 diabetes mellitus. Clin Endocrinol (Oxf) 2017; 87: 156-164
[39] Togashi Y, Miyamoto Y. Urinary cystatin C as a biomarker for diabetic nephropathy and its immunohistochemical localization in kidney in Zucker diabetic fatty (ZDF) rats. Exp Toxicol Pathol 2013; 65: 615-622

[40] Wanner C, Inzucchi SE, Lachin JM et al. Empagliflozin and progression of kidney disease in type 2 diabetes. N Engl J Med 2016; 375: 323-334 